\title{
Interstellar dust in the solar system: model versus in situ spacecraft data
}

\author{
Harald Krüger $^{1}$, Peter Strub ${ }^{1,2}$, Nicolas Altobelli ${ }^{3}$, Veerle J. Sterken ${ }^{4}$, \\ Ralf Srama ${ }^{2}$, and Eberhard Grün ${ }^{5}$
}

\author{
${ }^{1}$ Max-Planck-Institut für Sonnensystemforschung, Göttingen, Germany \\ e-mail: krueger@mps.mpg.de \\ ${ }^{2}$ Institut für Raumfahrtsysteme, Universität Stuttgart, Germany \\ ${ }^{3}$ European Space Agency, ESAC, Madrid, Spain \\ ${ }^{4}$ Institute of Applied Physics, University of Bern, Switzerland \\ ${ }^{5}$ Max-Planck-Institut für Kernphysik, Heidelberg, Germany
}

Received 25 September 2018 / Accepted 1 March 2019

\begin{abstract}
Context. In the early 1990s, contemporary interstellar dust penetrating deep into the heliosphere was identified with the in situ dust detector on board the Ulysses spacecraft. Later on, interstellar dust was also identified in the data sets measured with dust instruments on board Galileo, Cassini, and Helios. Ulysses monitored the interstellar dust stream at high ecliptic latitudes for about 16 yr. The three other spacecraft data sets were obtained in the ecliptic plane and cover much shorter time intervals.

Aims. To test the reliability of the model predictions, we compare previously published in situ interstellar dust measurements, obtained with these four spacecraft, with predictions of an advanced model for the dynamics of interstellar dust in the inner solar system (Interplanetary Meteoroid environment for EXploration; IMEX).

Methods. Micrometer and sub-micrometer-sized dust particles are subject to solar gravity, radiation pressure and the Lorentz force on a charged dust particle moving through the interplanetary magnetic field. These forces lead to a complex size-dependent flow pattern of interstellar dust in the planetary system. The IMEX model was calibrated with the Ulysses interstellar dust measurements and includes these relevant forces. We study the time-resolved flux and mass distribution of interstellar dust in the solar system.

Results. The IMEX model agrees with the spacecraft measurements within a factor of $2-3$, including time intervals and spatial regions not covered by the original model calibration with the Ulysses data set. The model usually underestimates the dust fluxes measured by the space missions which were not used for the model calibration, i.e. Galileo, Cassini, and Helios.

Conclusions. A unique time-dependent model, IMEX is designed to predict the interstellar dust fluxes and mass distributions for the inner and outer solar system. The model is suited to study dust detection conditions for past and future space missions.
\end{abstract}

Key words. zodiacal dust - dust, extinction - interplanetary medium - meteorites, meteors, meteoroids - ISM: abundances

\section{Introduction}

Interstellar dust became a topic of astrophysical research in the early 1930s when astronomers realized the extinction of starlight in the interstellar medium (ISM). At that time, information about dust in the ISM could only be obtained by astronomical observations. With the advent of dust detectors on board spacecraft in the 1970s, it became possible to investigate dust particles in situ, and the analysis of data obtained with the dust instruments flown on a couple of spacecraft suggested that interstellar dust can cross the heliospheric boundary and penetrate deep into the heliosphere (Bertaux \& Blamont 1976; Wolf et al. 1976, see Krüger \& Grün 2009 for a review). Later on, in the 1990s, this was undoubtedly demonstrated by the Ulysses spacecraft: the Ulysses dust detector, which measured mass, speed, and approach direction of the impacting particles, identified interstellar particles that have radii above $0.1 \mu \mathrm{m}$ sweeping through the heliosphere (Grün et al. 1993, 1994, 1995). These particles originated from the local interstellar cloud (LIC) surrounding our solar system (Frisch et al. 1999), thus providing an opportunity to probe dust from the LIC.

The motion of the heliosphere with respect to this cloud causes an inflow of interstellar dust into the heliosphere from a direction of $259^{\circ}$ ecliptic longitude and $8^{\circ}$ latitude (Landgraf 1998; Frisch et al. 1999; Strub et al. 2015) with an inflow speed of $26 \mathrm{~km} \mathrm{~s}^{-1}$ (Grün et al. 1994; Krüger et al. 2015). Within the measurement accuracy, the average dust inflow direction is coaligned with the interstellar neutral helium flow (Witte et al. 1996, 2004; Wood et al. 2015) ${ }^{1}$. The interstellar dust flow persists at high ecliptic latitudes above and below the ecliptic plane and even over the poles of the Sun, whereas interplanetary dust is strongly depleted at high latitudes (Grün et al. 1997).

The Ulysses interstellar dust measurements were confirmed by the Galileo (Baguhl et al. 1996; Altobelli et al. 2005a) and Cassini spacecraft (Altobelli et al. 2003, 2007, 2016), and interstellar impactors were also identified in the Helios dust data (Altobelli et al. 2006). In 2006, the Stardust mission successfully brought a sample of collected interstellar particles to Earth (Westphal et al. 2014). Finally, measurements by the radio and plasma wave instruments on board the STEREO and WIND

\footnotetext{
1 Working values of a speed of $25.4 \mathrm{~km} \mathrm{~s}^{-1}$ with directions from $255.7^{\circ}$ ecliptic longitude and $+5.1^{\circ}$ ecliptic latitude were suggested from Energetic Neutral Atom measurements by the Interstellar Boundary EXplorer mission (IBEX) by McComas et al. (2015) and recently confirmed by Swaczyna et al. (2018).
} 
spacecraft were explained by interstellar dust (Belheouane et al. 2012; Malaspina \& Wilson 2016), although this interpretation was recently called into question (Kellogg et al. 2018).

Measurements of interstellar dust inside the planetary system now provide a window for the study of solid interstellar matter at our doorstep (Frisch et al. 1999). However, the flow of the interstellar particles in the heliosphere is governed by two fundamental effects: the combined gravitational force and radiation pressure force of the Sun, and the Lorentz force acting on a charged particle moving through the solar magnetic field "frozen" into the solar wind. The former effect can be described as a multiplication of the gravitational force by a constant factor $(1-\beta)$, where the radiation pressure factor $\beta=\left|\mathbf{F}_{\text {rad }}\right| /\left|\mathbf{F}_{\text {grav }}\right|$ is a function of particle composition, size, and morphology. Interstellar particles approach the Sun on hyperbolic trajectories, leading to either a radially symmetric focussing $(\beta<1)$ or defocussing $(\beta>1)$ downstream of the Sun which is constant in time (Bertaux \& Blamont 1976; Landgraf 2000; Sterken et al. 2012). Particle sizes observed by the Ulysses dust detector typically range from approximately $0.1 \mu \mathrm{m}$ to several micrometers, corresponding to $0 \lesssim \beta \lesssim 1.9$ (Kimura et al. 2003; Landgraf et al. $1999)^{2}$. A detailed description of the forces acting on the particles and the resulting general interstellar dust flow characteristics was given by Sterken et al. (2012). Reviews about interstellar dust measurements in the solar system were recently given by Mann (2010) and Sterken et al. (2019).

The interplanetary magnetic field (IMF) shows systematic variations with time, including the 25-day solar rotation and the 22-yr solar magnetic cycle, as well as local deviations caused by disturbances in the IMF, due to for example coronal mass ejections (CMEs). The dust particles in interplanetary space are typically charged to an equilibrium potential of $+5 \mathrm{~V}$ (Kempf et al. 2004). Small particles have a higher charge-to-mass ratio, hence their dynamics is more sensitive to the IMF. The major effect of the magnetic field on the charged interstellar dust is a focussing and defocussing relative to the solar equatorial plane with the 22-yr magnetic cycle of the Sun (Landgraf 2000; Landgraf et al. 2003; Sterken et al. 2012, 2013). Modifications of the particle dynamics by solar radiation pressure and the Lorentz force acting on charged dust particles have to be taken into account for a proper interpolation of the interstellar dust properties to the ISM outside the heliosphere where these particles originate from.

Results of interstellar dust measurements and simulations (including mass distributions) from Galileo and Ulysses were compared and studied by Landgraf et al. (2000). A first comparison of the interstellar dust data obtained with four spacecraft, i.e. Ulysses, Galileo, Cassini, and Helios, was performed by Altobelli et al. (2005b). The results showed a decrease of the measured flux in the inner solar system which was attributed to heliospheric filtering. However, no comparison with a detailed dynamical model for all four missions was possible at the time. Detailed comparisons have only been made for the Galileo and Ulysses (partial) data sets (Landgraf 1998) and the (complete) Ulysses data set (Sterken et al. 2015). In this work we use these same data and compare them with simulation results obtained from our latest model for the dynamics of interstellar dust in the solar system (Landgraf 2000; Sterken et al. 2013; Strub et al. 2019).

\footnotetext{
2 Landgraf et al. (1999) found a range between $1.4<\beta<1.8$ from Ulysses measurements, and Kimura et al. (2003) found values for $\beta$ between 0 and 1.9 .
}

In Sect. 2 we briefly present the interstellar dust measurements obtained by the Helios, Cassini, Galileo, and Ulysses missions. A comprehensive description of the data analysis, in particular the identification scheme for the interstellar impactors, are provided in the publications by Altobelli et al. (2003, 2005a, 2006, 2016) for Helios, Galileo, and Cassini, and Strub et al. (2015) for the Ulysses data. In Sect. 3, we present our modelling results and compare these findings with the in situ measurements. Section 4 is a discussion and in Sect. 5 we summarize our conclusions.

\section{In situ spacecraft dust data}

The physical mechanism most generally utilized in modern space-borne in situ dust detectors is based on the measurement of the electric charge generated upon impact of a fast projectile on to a solid target (impact ionization). This yields the highest sensitivity for the detection of dust particles in space (Fechtig et al. 1978; Auer 2001). The impact can be detected by several independent measurements on various instrument channels (multi-coincidence detection) which allows for a reliable dust impact detection and identification of noise events (Grün et al. 1992a). The electrical charge generated upon impact can be empirically calibrated to provide the impact speed and mass of the particle (Göller \& Grün 1985). In combination with a time-of-flight mass spectrometer, an impact ionization detector can measure the chemical composition of the impacting particle (Srama et al. 2004).

In this work we use dust data obtained by impact ionization dust detectors on board the spacecraft Helios, Galileo, Cassini, and Ulysses (Dietzel et al. 1973; Grün 1981; Grün et al. 1992a,b; Srama et al. 2004). Impacts of interstellar dust particles in these data sets were identified by Altobelli et al. (2006, 2005a, 2003, 2016) and Strub et al. (2015). We do not consider dust measurements with other detection techniques because we want to keep the data set as consistent as possible. Different detection techniques are usually connected with individual systematic uncertainties, for example due to mass calibration or instrument detection threshold, increasing the overall uncertainty.

When a dust particle strikes a solid target with high speed $\left(\gg 1 \mathrm{~km} \mathrm{~s}^{-1}\right)$, it forms a crater in the target and releases ejecta composed of both particle and target material. The ejecta consist of positive and negative ions, electrons, and neutral atoms and molecules originating from both projectile and target. Because of its high internal pressure (up to $5 \mathrm{TPa}$ ), the ejecta cloud expands rapidly into the surrounding vacuum.

The sensors consist of a metal plate target and a collector (e.g. a metal grid) for either the ions or electrons of the impact plasma. Different electric potentials applied to the target plate and the collector generate an electric field, separating the positively and negatively charged ions. Charge-sensitive amplifiers coupled to both the target plate and the collector register independently, but simultaneously, an impacting dust particle. The total amount of charge, $Q_{\mathrm{imp}}$, collected on each channel is a function of mass $m_{\mathrm{d}}$ and impact speed $v_{\mathrm{d}}$ of the particle as well as the composition of the particle. The value $Q_{\text {imp }}$ can be described by the empirical law

$Q_{\text {imp }}=K m_{\mathrm{d}}^{\alpha} v_{\mathrm{d}}^{\gamma}$,

where $\alpha \simeq 1$ and $1.5 \lesssim \gamma \lesssim 5.5$ in the speed range $2 \mathrm{~km} \mathrm{~s}^{-1}$ $\lesssim v \lesssim 70 \mathrm{~km} \mathrm{~s}^{-1}$ (Auer 2001; Stübig 2002). The value $K$ depends on the sensor geometry and the signal processing by the instrument electronics. In particular, for constant impact speed, the 
Table 1. Characteristics of the individual spacecraft measurements.

\begin{tabular}{|c|c|c|c|c|c|c|c|c|c|c|}
\hline \multirow{2}{*}{$\begin{array}{l}\text { Mission/ } \\
\text { interval }\end{array}$} & \multirow{2}{*}{$\begin{array}{l}\text { Start time } \\
\text { (year-doy) } \\
\quad(2)\end{array}$} & \multirow{2}{*}{$\begin{array}{l}\text { End time } \\
\text { (year-doy) } \\
\quad(3)\end{array}$} & \multirow{2}{*}{$\begin{array}{l}\text { Range } \\
(\mathrm{AU}) \\
(4)\end{array}$} & \multirow{2}{*}{$\begin{array}{c}\text { Impact } \\
\text { speed } \\
v_{\mathrm{d}} \\
\left(\mathrm{km} \mathrm{s}^{-1}\right) \\
(5)\end{array}$} & \multirow{2}{*}{$\begin{array}{l}Q_{\mathrm{imp}} / m_{\mathrm{d}}(d) \\
\left(\mathrm{C} \mathrm{kg}^{-1}\right) \\
(6)\end{array}$} & \multicolumn{3}{|c|}{ Detection threshold $^{(a)}$} & \multirow{2}{*}{$\begin{array}{l}N^{(e)} \\
(10)\end{array}$} & \multirow{2}{*}{$\begin{array}{l}\text { Average flux } \\
\qquad(e) \\
\left(\mathrm{m}^{-2} \mathrm{~s}^{-1}\right) \\
(11)\end{array}$} \\
\hline & & & & & & $\begin{array}{c}\text { Charge } \\
Q_{\text {imp }}(\mathrm{C}) \\
\quad(7)\end{array}$ & $\begin{array}{c}\text { Mass } \\
m_{\mathrm{d}}(\mathrm{kg}) \\
(8)\end{array}$ & $\begin{array}{c}\text { Radius } \\
r_{\mathrm{d}}(\mu \mathrm{m}) \\
\quad(9)\end{array}$ & & \\
\hline $\begin{array}{l}\text { Helios } \\
\text { HEL }^{(b)}\end{array}$ & $1974-353$ & 1980-002 & $0.3-1.0$ & 60 & $2.6 \times 10^{3}$ & $2 \times 10^{-12}$ & $7 \times 10^{-16}$ & 0.37 & 27 & $(2.6 \pm 0.3) \times 10^{-6}$ \\
\hline $\begin{array}{l}\text { Galileo } \\
\text { GLL1 }\end{array}$ & 1990-001 & 1990-190 & $0.7-1.2$ & 50 & $5.0 \times 10^{4}$ & $2 \times 10^{-12}$ & $4 \times 10^{-17}$ & 0.14 & 21 & $(7.0 \pm 1.5) \times 10^{-5}$ \\
\hline $\begin{array}{l}\text { GLL2 } \\
\text { GLL3 } \\
\text { GLL4 } \\
\text { GLL5 }\end{array}$ & $\begin{array}{l}1991-056 \\
1991-228 \\
1993-005 \\
1993-182\end{array}$ & $\begin{array}{l}1991-123 \\
1991-340 \\
1993-181 \\
1993-365\end{array}$ & $\begin{array}{l}1.0-1.4 \\
1.9-2.2 \\
1.2-2.5 \\
2.5-3.5\end{array}$ & $\begin{array}{l}50 \\
30 \\
50 \\
35\end{array}$ & $\begin{array}{l}5.0 \times 10^{4} \\
6.5 \times 10^{3} \\
5.0 \times 10^{4} \\
1.1 \times 10^{4}\end{array}$ & $\begin{array}{l}2 \times 10^{-12} \\
1 \times 10^{-13} \\
2 \times 10^{-12} \\
1 \times 10^{-13}\end{array}$ & $\begin{array}{l}4 \times 10^{-17} \\
2 \times 10^{-17} \\
4 \times 10^{-17} \\
9 \times 10^{-18}\end{array}$ & $\begin{array}{l}0.14 \\
0.11 \\
0.14 \\
0.09\end{array}$ & $\begin{array}{l}13 \\
19 \\
22 \\
41\end{array}$ & $\begin{array}{l}(9.5 \pm 1.5) \times 10^{-5} \\
(3.5 \pm 0.8) \times 10^{-5} \\
(8.0 \pm 1.0) \times 10^{-5}\end{array}$ \\
\hline $\begin{array}{l}\text { Cassini } \\
\text { CAS1 } \\
\text { CAS2 }\end{array}$ & $\begin{array}{l}1999-081 \\
2004-183\end{array}$ & $\begin{array}{l}1999-181 \\
2013-364\end{array}$ & $\begin{array}{l}0.7-1.2 \\
9.1-9.9\end{array}$ & $\begin{array}{l}45 \\
30\end{array}$ & $\begin{array}{l}6.0 \times 10^{4} \\
8.0 \times 10^{3}\end{array}$ & $\begin{array}{l}3 \times 10^{-12} \\
1 \times 10^{-15}\end{array}$ & $\begin{array}{l}5 \times 10^{-17} \\
5 \times 10^{-18}\end{array}$ & $\begin{array}{l}0.15 \\
0.07\end{array}$ & $\begin{array}{l}14 \\
36\end{array}$ & $\begin{array}{l}(2.5 \pm 0.5) \times 10^{-5} \\
(1.5 \pm 0.5) \times 10^{-4}\end{array}$ \\
\hline $\begin{array}{l}\text { Ulysses } \\
\text { ULS1 } \\
\text { ULS2 } \\
\text { ULS3 } \\
\text { ULS4 } \\
\text { ULS5 }\end{array}$ & $\begin{array}{l}1992-245 \\
1995-166 \\
1996-131 \\
2002-131 \\
2005-245\end{array}$ & $\begin{array}{l}1994-131 \\
1996-131 \\
2000-131 \\
2002-363 \\
2006-245\end{array}$ & $\begin{array}{l}3.0-5.0 \\
1.9-3.7 \\
3.7-5.4 \\
3.5-4.4 \\
3.2-4.9\end{array}$ & $\begin{array}{l}30 \\
30 \\
30 \\
25 \\
30\end{array}$ & $\begin{array}{l}6.5 \times 10^{3} \\
6.5 \times 10^{3} \\
6.5 \times 10^{3} \\
4.0 \times 10^{3} \\
6.5 \times 10^{3}\end{array}$ & $\begin{array}{l}1 \times 10^{-13} \\
1 \times 10^{-13} \\
1 \times 10^{-13} \\
1 \times 10^{-13} \\
1 \times 10^{-13}\end{array}$ & $\begin{array}{l}2 \times 10^{-17} \\
2 \times 10^{-17} \\
2 \times 10^{-17} \\
3 \times 10^{-17} \\
2 \times 10^{-17}\end{array}$ & $\begin{array}{l}0.11 \\
0.11 \\
0.11 \\
0.13 \\
0.11\end{array}$ & $\begin{array}{l}116 \\
39 \\
94 \\
37 \\
79\end{array}$ & $\begin{array}{l}(7.7 \pm 2.0) \times 10^{-5} \\
(5.3 \pm 1.7) \times 10^{-5} \\
(2.9 \pm 1.1) \times 10^{-5} \\
(1.1 \pm 0.3) \times 10^{-4} \\
(1.1 \pm 0.2) \times 10^{-4}\end{array}$ \\
\hline
\end{tabular}

Notes. Spacecraft (Col. 1), measurement periods (Cols. 2 and 3), heliocentric distance range (Col. 4), average interstellar dust impact speed derived from the model (Col. 5), charge-to-mass ratio from instrument calibration (Col. 6), detection thresholds (Cols. 7 to 9), number of identified interstellar particles (Col. 10), and average interstellar dust fluxes (Col. 11). ${ }^{(a)}$ Detection threshold based on the identification scheme for interstellar particles. Throughout this paper we calculate particle radii from the measured masses by assuming a spherical particle shape and a density typical of astronomical silicates $\rho_{\mathrm{d}}=3300 \mathrm{~kg} \mathrm{~m}^{-3}$ (Kimura \& Mann 1999). The particle radius is given by

$r_{\mathrm{d}}=\sqrt[3]{\frac{3 m_{\mathrm{d}}}{4 \pi \rho_{\mathrm{d}}}}$,

where $m_{\mathrm{d}}$ is the dust particle mass derived from the instrument calibration. ${ }^{(b)}$ For Helios we considered only impacts when the true anomaly angle of the spacecraft was in the range $-180^{\circ}<v<90^{\circ}$ consistent with Altobelli et al. (2006). ${ }^{\left({ }^{c}\right)}$ Cassini CDA was not pointing towards the direction of interstellar dust continuously during this time interval (Altobelli et al. 2016).

References. ${ }^{(d)}$ Grün (1981); Grün et al. (1995); Stübig (2002). ${ }^{(e)}$ Altobelli et al. (2003, 2005a, 2006), Strub et al. (2015).

charge generated upon impact is proportional to the particle mass (Göller \& Grün 1985).

The instrument sensitivity, expressed by the parameter $K$, is determined by the technical detection threshold for the impact charge measurement, which is about $10^{-14} \mathrm{C}$ for Ulysses, Galileo, and Helios, and $10^{-15} \mathrm{C}$ for Cassini, respectively. However, the interstellar impactors had to be separated from interplanetary particle impacts which was usually done by their impact direction and impact speed (or generated impact charge). This led to specific identification criteria for the interstellar particles in the data sets of the four space missions, and thus in most cases to less sensitive detection thresholds than the technically detectable threshold. The measurement periods of the different space missions, details of the particle identification schemes and derived dust fluxes are summarized in Table 1.

The particle speed can be determined from the rise times of the individual charge signals (Göller \& Grün 1989). For a given impact speed the signal strength also moderately depends on the particle material and on the impact angle. Neither the particle material nor the impact angle are known for an impinging particle. Therefore, averaged calibration curves have to be used to obtain impact speeds, assuming that the materials used for calibration represent cosmic dust particles (Grün et al. 1995). The typical accuracy of the derived speed $v_{\mathrm{d}}$ is a factor of 2 .

Once the particle speed has been determined, its chargeto-mass ratio $Q_{\mathrm{imp}} / m_{\mathrm{d}}$ generated upon impact can be derived from empirical calibration curves. These were obtained from impact experiments at the dust accelerator facility at MaxPlanck-Institut für Kernphysik, Heidelberg ( $\gamma$ in Eq. (1); Grün 1981; Grün et al. 1995; Stübig 2002; Srama et al. 2004; Srama 2009). In the next step, the particle mass can be derived from the calibrated impact charge-to-mass ratio and the measured impact charges. If the speed is well determined, the mass can also be derived with a higher accuracy. The typical uncertainty in the derived mass $m_{\mathrm{d}}$ is a factor of 10 .

Given that the charge-to-mass ratio $Q_{\mathrm{imp}} / m_{\mathrm{d}}$ strongly depends on particle impact speed, we had to assume a speed to derive the particle mass from the measured impact charge in the spacecraft data (Eq. (1)). For our analysis we took average particle speeds from the model for the measurement time interval considered (Sect. 3), and the corresponding $Q_{\text {imp }} / m_{\mathrm{d}}$ listed in Table 1 . 


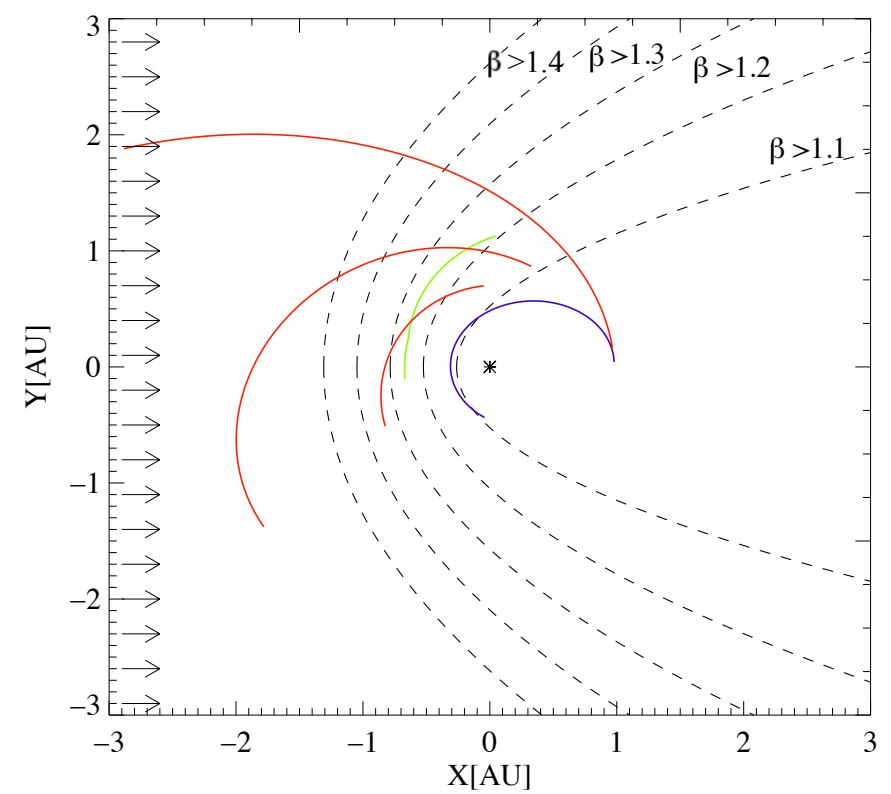

Fig. 1. Trajectory segments of Helios (blue), Galileo (red), and Cassini (green) favourable for the identification of interstellar dust. The Sun is at the centre and the $X-Y$ plane is the ecliptic plane. Vernal equinox is approximately towards the $Y$-direction, and the nominal interstellar dust flow direction is indicated by arrows. Different $\beta$ avoidance cones are indicated by dashed lines. See text for details.

\subsection{Helios}

The Helios 1 spacecraft (we refer only to Helios 1 throughout this paper) was launched into a heliocentric orbit on 10 December 1974, with perihelion and aphelion distances of 0.3 and 1.0 AU, respectively (Fig. 1). The spacecraft was spinstabilized with a spin axis pointing normal to the ecliptic plane and a spin period of one second. It carried two dust instruments: the ecliptic sensor which was exposed to sunlight and the south sensor which was shielded by the spacecraft from direct sunlight (Dietzel et al. 1973; Fechtig et al. 1978; Grün 1981; Altobelli et al. 2006).

Between 19 December 1974 and 2 January 1980 the Helios sensors transmitted the data of 235 dust impacts to Earth (Grün 1981). Interstellar impactors could only be separated from interplanetary dust particles if their impact charge exceeded $2 \times 10^{-12} \mathrm{C}$ and only during limited periods of the Helios orbit. This led to the identification of 27 interstellar impactors during ten orbits of Helios around the Sun when the true anomaly angle $v$ of the spacecraft was in the range $-180^{\circ}<v<90^{\circ}$ (Altobelli et al. 2006). Calibration parameters $K=4.07 \times 10^{-5}$ (mass taken in gram) and $\gamma=2.7$ (Grün 1981) were used, and the derived particle masses were mostly in the range $10^{-15} \mathrm{~kg} \lesssim m_{\mathrm{d}} \lesssim 10^{-14} \mathrm{~kg}$. Details of the Helios measurements are summarized in Table 1.

In addition to measuring particle masses and fluxes, the instruments performed a low-resolution compositional analysis with a time-of-flight analyser (Auer 2001). The Helios dust analysers were the first instruments measuring the elemental composition of dust particles in interplanetary space.

\subsection{Galileo}

Galileo was launched on 18 October 1989, and the spacecraft had enough energy to reach Jupiter in December 1995 after two fly-bys at Earth and one at Venus. Galileo was the first spacecraft in orbit about Jupiter until the mission was terminated on 21 September 2003. Galileo was a dual-spinning spacecraft; the dust detector was mounted on the despun section of the spacecraft. The Galileo dust detector measured dust particle flux, impact direction, speed, and mass of the impacting particles (Grün et al. 1992a). It was a twin of the dust detector on board Ulysses (Grün et al. 1992b).

During the interplanetary mission of Galileo three orbit segments had a detection geometry which allowed the identification of interstellar dust (Fig. 1). Owing to the varying impact speeds, different charge detection thresholds apply to these intervals (corresponding to impact charge thresholds ranging from $1 \times 10^{-13} \mathrm{C}$ to $2 \times 10^{-12} \mathrm{C}$; cf. Table 1$)$. A total of 115 interstellar impactors were identified in the Galileo data set (Altobelli et al. 2005a). For our analysis we have split the three orbit segments shown in Fig. 1 into five time intervals. The mass calibration was obtained from an empirical calibration curve (Grün et al. 1995, their Fig. 3a).

\subsection{Cassini}

The Cassini spacecraft was launched on 15 October 1997. During its first two years in interplanetary space the spacecraft performed two fly-bys at Venus and one at Earth to gain enough energy to reach Saturn. In 2004 it became the first spacecraft in orbit about the giant ring planet, until the mission was terminated on 15 September 2017. Cassini was a three-axis stabilized spacecraft.

The Cassini Cosmic Dust Analyzer (CDA) was an upgrade of the dust detectors flown on board Galileo and Ulysses, measuring particle composition and electric charge in addition to particle mass, impact speed, flux, and direction (Srama et al. 2004). For CDA we used the mass calibration derived by Stuibig (2002, his Fig. 5.1).

As a consequence of operational constraints of the Cassini spacecraft during its interplanetary voyage, interstellar dust particles could only be measured during approximately three months from 22 March 1999 to 30 June 1999 at a heliocentric distance between 0.7 and 1.2 AU (Fig. 1). A charge detection threshold of $3 \times 10^{-12} \mathrm{C}$ had to be used in this period, and $14 \pm 3$ particle impacts of likely interstellar origin were identified in the mass range $5 \times 10^{-17} \mathrm{~kg} \leq m_{\mathrm{d}} \leq 10^{-15} \mathrm{~kg}$ (Altobelli et al. 2003). Details of the Cassini measurements can be found in Table 1.

The Cassini CDA instrument also detected interstellar dust in the Saturnian system (Altobelli et al. 2016, Fig. 2). A total of 36 interstellar particles were identified within a distance range of 9-60 Saturn radii from the planet by their high entry speed into the Saturnian system and their impact direction which was compatible with the expected interstellar dust flow direction at Saturn. The derived average particle flux was $1.5 \times 10^{-4} \mathrm{~m}^{-2} \mathrm{~s}^{-1}$ in the mass range $5 \times 10^{-18} \mathrm{~kg} \leq m_{\mathrm{d}} \leq 5 \times 10^{-16} \mathrm{~kg}$.

Impacts on to the CDA Chemical Analyzer Target (CAT) by sufficiently large particles do not provide time-of-flight spectra with sufficiently well-resolved spectral lines from which the minimum impact speed can be derived. The Cassini 2 measurement interval therefore lacks particles heavier than approximately $5 \times 10^{-16} \mathrm{~kg}$.

From the rise time of the impact charge signals the particle impact speed can usually be determined with a factor of 2 uncertainty. The shape of the time-of-flight mass spectra produced by the CDA CAT, however, provides a more accurate determination of the minimum impact speed of each impactor. This method, because it is based on the velocity-mass calibration for the CAT, therefore also provides a better estimate of the upper mass value for each impactor (compared to the factor of ten; see Sect. 2 above). The time-of-flight mass spectra method also yields a 


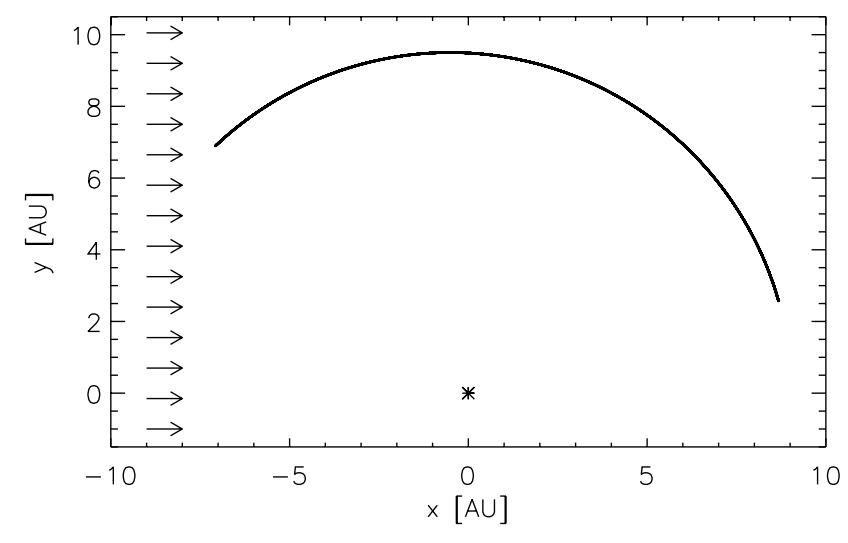

Fig. 2. Same as Fig. 1 but for Cassini at Saturn.

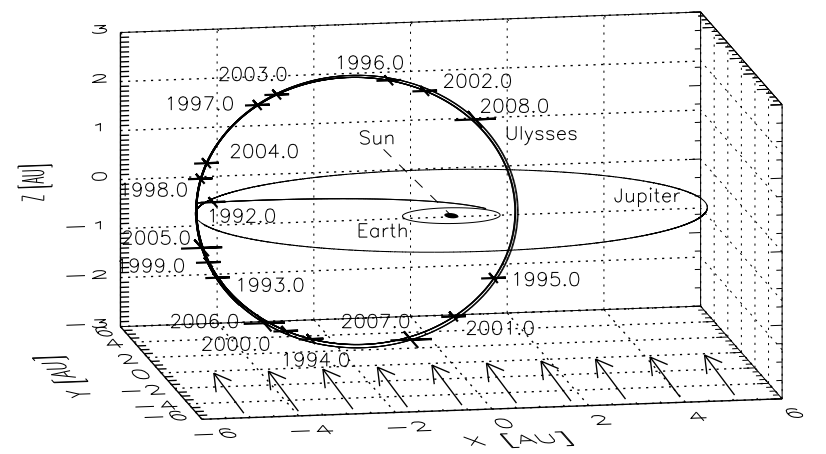

Fig. 3. Trajectory of Ulysses in ecliptic coordinates with the Sun at the centre. The orbits of Earth and Jupiter indicate the ecliptic plane, and the initial trajectory of Ulysses was in this plane. After Jupiter fly-by in early 1992 , the orbit was almost perpendicular to the ecliptic plane $\left(79^{\circ}\right.$ inclination). Crosses denote the spacecraft position at the beginning of each year. Vernal equinox is to the right (positive $x$-axis). Arrows indicate the nominal interstellar dust flow direction, which is within the measurement accuracy co-aligned with the direction of the interstellar helium gas flow. It is almost perpendicular to the orbital plane of Ulysses.

lower dust detection statistics, compared to measurements performed by the dust instruments on board Ulysses and Galileo, because of the smaller CAT surface.

\subsection{Ulysses}

Ulysses was launched on 6 October 1990. During a fly-by at Jupiter on 8 February 1992 this spacecraft was deflected on to an orbit almost perpendicular to the ecliptic plane and it became the first spacecraft on a polar orbit about the Sun. Operations of Ulysses were terminated on 29 June 2009. The dust detector on board (Grün et al. 1992b) was a twin of the Galileo dust detector. Ulysses was a spinning spacecraft with a period of five revolutions per minute. The Ulysses trajectory is shown in Fig. 3.

The Ulysses mission was particularly well suited for the detection of interstellar particles. First, its highly inclined orbit with an aphelion at approximately Jupiter's orbit (5.5 AU) took the spacecraft far above the ecliptic plane. Given that the concentration of interplanetary dust particles drops at increasing ecliptic latitudes and that most of the interplanetary dust moves on prograde heliocentric orbits, the orbital sections where Ulysses was far from its perihelion and far from the solar poles were best suited for the detection of interstellar dust. The orientation of the orbital ellipse of Ulysses was such that in these sections the impact directions of interplanetary and interstellar particles were almost antiparallel, and thus these populations could easily be separated.

The Ulysses dust data set is by far the largest of in situ interstellar dust measurements available to date. A detection threshold of $1 \times 10^{-13} \mathrm{C}$ had to be used and the data set contains more than 900 identified interstellar particles, covering about $75 \%$ of one full 22-yr solar cycle (Strub et al. 2015; Krüger et al. 2010, 2015). For our analysis we selected five mission intervals when the dust detector was continuously measuring dust (cf. Table 1). Similar to Galileo, the mass calibration was obtained from an empirical calibration curve (Grün et al. 1995, their Fig. 3a). Given the long time coverage and thus large number of identified interstellar particles, the dust model we use in Sect. 3 was calibrated with the Ulysses interstellar dust data set (Strub et al. 2019).

\section{Interstellar dust simulations}

Previous models for interstellar dust in the solar system described the interstellar dust flow at larger heliocentric distances well, but they did not have the resolution to enable a good time-resolved description of the dust environment at Earth (Grün et al. 1994; Landgraf 2000; Sterken et al. 2012). Based on these earlier models and the dust measurements by the Ulysses spacecraft, Strub et al. (2019) executed high-resolution simulations in the context of the IMEX modelling effort (Interplanetary Meteoroid environment for EXploration), which included an interstellar dust module developed for this purpose. The authors simulated the dynamics of charged micrometer and submicrometer-sized interstellar particles exposed to solar gravity, solar radiation pressure, and a time-varying IMF. The mass distribution is represented by 12 particle sizes between 0.049 and $4.9 \mu \mathrm{m}$, and the dynamics of each of these sizes was simulated individually.

In IMEX the dust density in the solar system is calibrated with the Ulysses interstellar dust measurements, which is by far the most comprehensive data set of interstellar dust measurements presently available (Strub et al. 2015; Krüger et al. 2015). Each particle size bin in the model was calibrated such that the average dust flux measured by Ulysses in this size bin was reproduced (Strub et al. 2019). Because of the variation of the IMF imposed by the 22-yr solar cycle, the model is time-dependent. Details of the model and general interstellar dust flow characteristics are provided in Sterken et al. (2012), and those of the flow at Earth orbit are found in Strub et al. (2019). We use IMEX to simulate dust fluxes and we compare the results with the dust measurements discussed in Sect. 2.

Throughout this paper we use the inflow direction of the interstellar dust into the heliosphere from a direction of $259^{\circ}$ ecliptic longitude and $8^{\circ}$ latitude and inflow speed of $26 \mathrm{~km} \mathrm{~s}^{-1}$ as the nominal direction and speed of the undisturbed interstellar dust flow. This is equivalent to the interstellar particles being at rest with respect to the LIC, and these particles approach the Sun on hyperbolic trajectories. The trajectories of particles with $\beta=1$ are altered neither by solar radiation nor by solar gravity. The only force leading to a deflection of these particles is the Lorentz force imposed by the IMF. For particles with $\beta \neq 1$ the solar radiation pressure leads to either a concentration of particles $(\beta<1)$ downstream of the Sun, or to a deflection $(\beta>1)$ and thus to a time-independent depletion cone downstream of the Sun (Fig. 1).

In our model, the dynamics of particles with approximate radii $0.1 \mu \mathrm{m} \lesssim r_{\mathrm{d}} \lesssim 0.5 \mu \mathrm{m}$ are dominated by solar radiation 
pressure, larger particles are dominated by gravity, and smaller particles by the Lorentz force imposed by the IMF. The $\beta$-mass relation used for each particle size in our simulations is determined according to the "adapted astronomical silicates $\beta$-curve" (Sterken et al. 2012), which combines the radiation pressure efficiencies of Gustafson (1994) with an average of the maximum values for $\beta$ from Ulysses observations $(\beta=1.6$; from Landgraf et al. 1999).

The time-variable IMF evolves through focussing and defocussing configurations during the $22-y r$ solar cycle. This causes time-dependent concentrations and rarefactions of interstellar particles smaller than approximately $0.3 \mu \mathrm{m}$ in the inner solar system. We use the solar cycle approximation used by Strub et al. (2019, their Fig. 1). Around the solar minima in 1974 and 1996, the IMF was in a defocussing configuration and around the solar minima in 1985 and 2007 the IMF was in a focussing configuration for these small particles.

We did not take into account the sensitivity profiles of the individual dust instruments. Instead, the model assumes a spherical sensor with $4 \pi$ sensitivity characteristics, i.e. all particles reaching the spacecraft are taken into account. Given that for all space missions under consideration the dust sensors had a rather wide field of view and the interstellar dust flow is rather collimated, this is a reasonable approximation.

\subsection{Dust size distributions}

The simulated interstellar dust size distributions for the measurement periods of the four spacecraft considered in this work are shown in Fig. 4. Vertical dashed lines indicate the detection thresholds which had to be applied to separate interstellar particles from other dust populations in the data sets (Altobelli et al. 2003, 2005a, 2006, 2016; Strub et al. 2015). Thus, interstellar particles to the left of the dashed lines - if present - could not be extracted from the data. Therefore, to avoid confusion in the following discussion, we do not show particles in this size range in Fig. 4 even though they may be present in the model.

Strong variations are imposed by the varying heliocentric distances of the spacecraft and the time-dependent IMF configuration. The smallest particles are most effectively prevented from entering the inner solar system during defocussing configurations of the IMF. This leads to a strong depletion of particles smaller than approximately $0.3 \mu \mathrm{m}$, in particular when the Helios and Cassini measurements were taken in the innermost regions of the solar system (cf. Table 1). Furthermore, particles in the size range $0.1 \mu \mathrm{m} \lesssim r_{\mathrm{d}} \lesssim 0.5 \mu \mathrm{m}$ are depleted by the solar radiation pressure. Similarly, the Ulysses measurements 2-5 were taken during the defocussing configuration and are thus also strongly depleted in small particles. On the other hand, the Galileo intervals 1-3 were in the focussing phase of the IMF when small particles could reach the inner solar system.

In Fig. 5 we compare the simulated dust mass distributions with the spacecraft measurements; the conversion between particle mass and radius is done with Eq. (2) (see footnote of Table 1). Again, the detection thresholds are indicated (Table 1, Col. 8). Particle masses were calculated from the measured impact charges with the average particle impact speeds derived from the simulations (Table 1, Col. 5), in the same way as the instrument detection thresholds.

In general, the masses predicted by the IMEX model are in good agreement with the measurements, only the Ulysses data show some impacts below the calculated detection thresholds. This is likely due to uncertainties in the mass calibration and/or the detection threshold. We note that particle masses were derived from the measured impact charge using Eq. (1) with a typical factor of 10 uncertainty in the mass calibration. Furthermore, all impacts were calibrated with the same average impact speed derived from the model, while the model predicts a speed variation of about a factor of two during each of the measurement intervals. For the second Cassini interval, the minimum impact speed was derived from the impact spectra with a typical accuracy of $5 \mathrm{~km} \mathrm{~s}^{-1}$, leading to a higher accuracy in the mass calibration. Thus, the data in Fig. 5 are binned in intervals with one order of magnitude bin width, except for Cassini 2 for which we achieved a higher mass resolution (cf. Sect. 2.3).

\subsection{Dust fluxes}

In Figs. 6 and 7 we show the simulated dust fluxes integrated over all detectable particle sizes and compare these fluxes with the measurements. We added up simulated fluxes for sizes only above the detection threshold shown in Fig. 4 because smaller particles were not detectable by the dust instruments. The simulations were performed for the same time intervals as the measurements (Cols. 2 and 3 in Table 1). All measured and simulated dust fluxes discussed in this paper are given in the heliocentric reference system.

The model predicts on average somewhat lower fluxes than measured. This is also the case for Ulysses, even though the Ulysses data were used to calibrate the IMEX model. We come back to this in Sect. 4. The mean value of the ratio of measured to simulated fluxes for Galileo, Cassini, and Helios is $1.8_{-0.9}^{+2.1}$; for all measurements including Ulysses the value is $2.0_{-1.0}^{+2.2}, 1 \sigma$ uncertainty.

\section{Discussion}

The IMEX model was calibrated with the Ulysses interstellar dust data set because it is the most comprehensive and homogeneous measurement by a single dust instrument over a period of $16 \mathrm{yr}$, covering a large portion of a full 22-yr solar cycle (Fig. 7). With more than 900 identified interstellar particles this model has the best statistical accuracy of all interstellar dust measurements with a dedicated dust detector obtained to date. For three of the five Ulysses measurement intervals the model reproduces the data within a factor of 2 . The discrepancy is somewhat more pronounced only after 2002.

Given that the Ulysses data set was used to calibrate the IMEX model, this discrepancy is surprising at first glance. However, the model was calibrated with the full Ulysses data set, and it reproduces the overall interstellar dust fluxes measured during the entire mission to within $2 \%$. This makes us very confident that the overall calibration of the model consistently reproduces the Ulysses measurements.

For the analysis in this paper we used five relatively short time intervals for Ulysses instead of the full mission data set, mainly for two reasons: we wanted to have a comparable number of dust impact events in each time interval as is the case for the other missions considered in this paper and we wanted to cover shorter heliocentric distance ranges than the entire Ulysses mission. Furthermore, we ignored the spatial region in the vicinity of Jupiter to make sure that we do not have a contamination by Jupiter stream particles (Krüger et al. 2006). Thus, we disregarded the time period from 2003 to mid 2005. It is not surprising that we get a somewhat larger discrepancy between model and data for shorter time intervals even though the model agrees very well with the data for the full time period. 

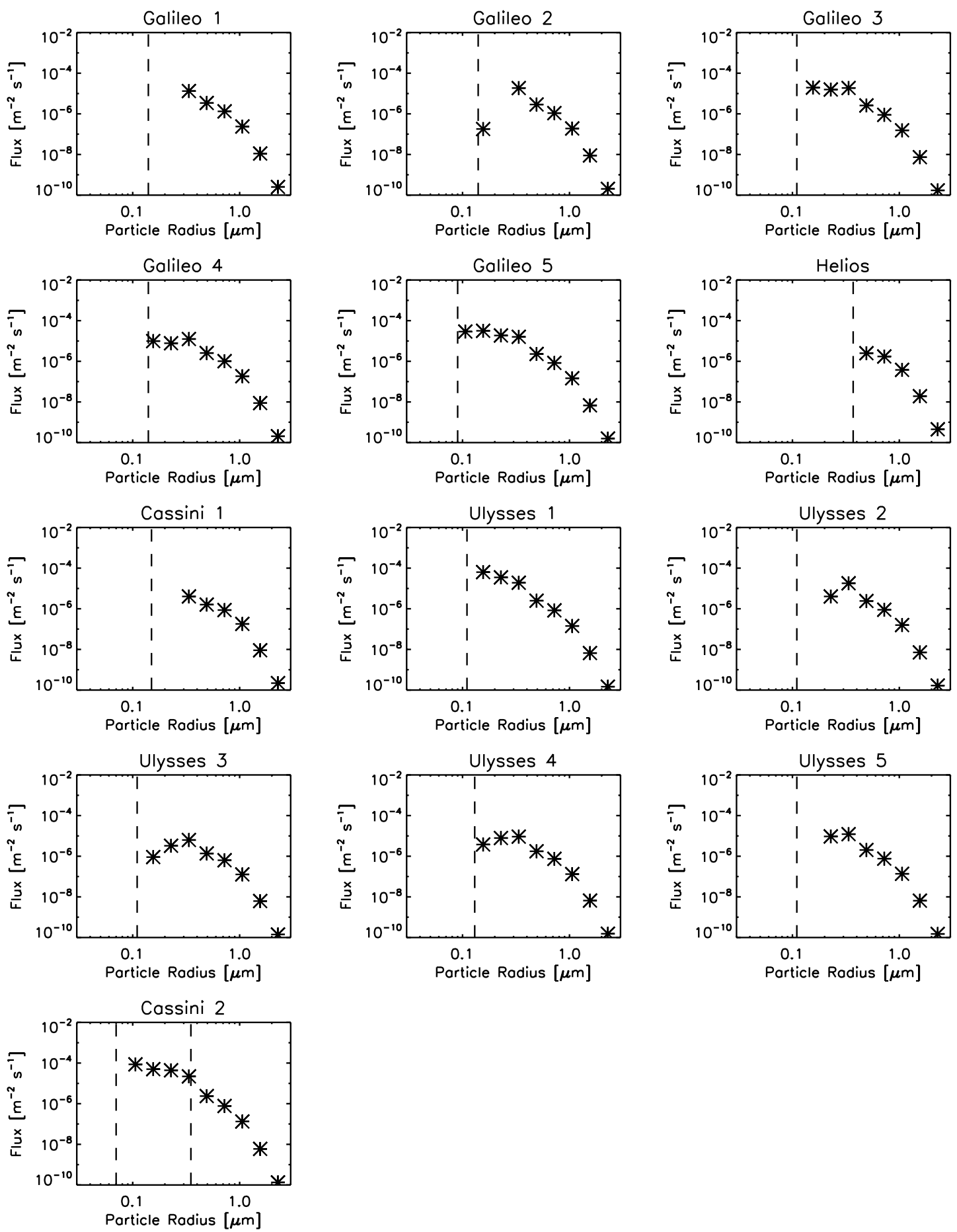

Fig. 4. Interstellar particle size distributions obtained from the simulations for the various missions and orbit segments given in Table 1. Dashed vertical lines indicate the detection thresholds of the dust instruments assuming an average particle impact speed derived from the model, as discussed in Sect. 2 (Cols. 5 and 9 in Table 1). For the Cassini 2 interval the upper detection limit at approximately $0.35 \mu$ m particle radius for the CDA CAT is also shown.

The IMEX model reproduces most of the Galileo, Helios, and Cassini measurements to within a factor of 2-3. It should be noted that the model as calibrated with the Ulysses data shows a tendency to predict lower fluxes than measured by the space instruments.

Furthermore, discrepancies between model and data may be related to the demonstration by Sterken et al. (2015) that either the first part of the data (before 2002) or the last part (2002-2008) can be well represented by simulations of one dust population (size and physical properties) but not both periods in one simulation with a single set of particle properties. In 2005 a rapid change in interstellar flow direction and density was seen in the Ulysses data (Krüger et al. 2007), while in 2006 the flow direction was again co-aligned with the nominal flow direction of the interstellar helium within the measurement accuracy (Strub et al. 2015). The authors concluded that this was a temporally limited phenomenon. Sterken et al. (2015) simulated this shift in dust direction (i.e. the data after 2002) for porous bigger particles 

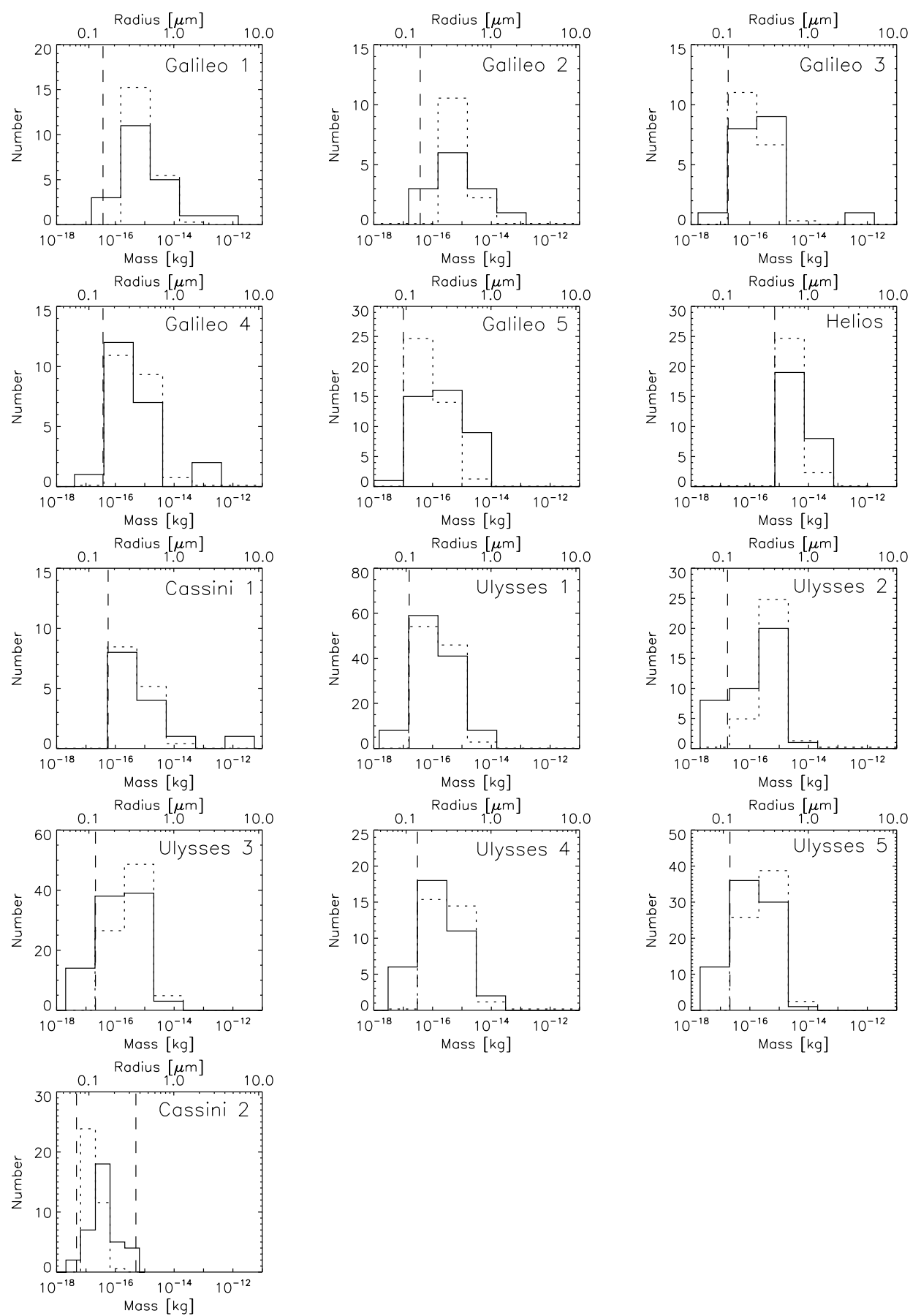

Fig. 5. Mass distributions of interstellar impactors derived from the instrument calibration with the calibration parameters listed in Table 1 (solid histograms; from Altobelli et al. 2005a, 2003, 2006, 2016; Strub et al. 2015). Dashed histograms show the fluxes shown in Fig. 4 derived from the model. The model curves are normalized such that they contain the same number of particles as measured by the spacecraft detector in the time interval under consideration. Vertical dashed lines indicate the detection threshold assuming an average particle impact speed derived from the model (Cols. 5 and 9 in Table 1); for the Cassini 2 interval the upper detection limit for the CDA CAT is also shown. Particle radii are indicated at the top.

$(\gtrsim 0.2 \mu \mathrm{m}$ radius $)$ and for rather compact smaller $(\lesssim 0.2 \mu \mathrm{m})$ particles. An extra (solar-cycle dependent) filtering mechanism in the outer boundary regions of the heliosphere (Kimura \& Mann 1998) included in the simulations may lead to a better fit for the entire Ulysses data set, in particular to reproduce the shift in dust flow direction in 2005.

The Ulysses measurements that were used for the model calibration covered the time period from 1992 to 2007, a spatial region between approximately 2 and $5 \mathrm{AU}$, and most of the time they were acquired far away from the ecliptic plane (Strub et al. 2015; Krüger et al. 2015). The measurements by the other spacecraft were obtained in the ecliptic plane at distance ranges much closer to the Sun inside Earth's orbit (Helios, Galileo and Cassini 1), further away from the Sun at Saturn (Cassini 2), or many years before Ulysses (Helios). The model shows overall good agreement with all these dust measurements, 


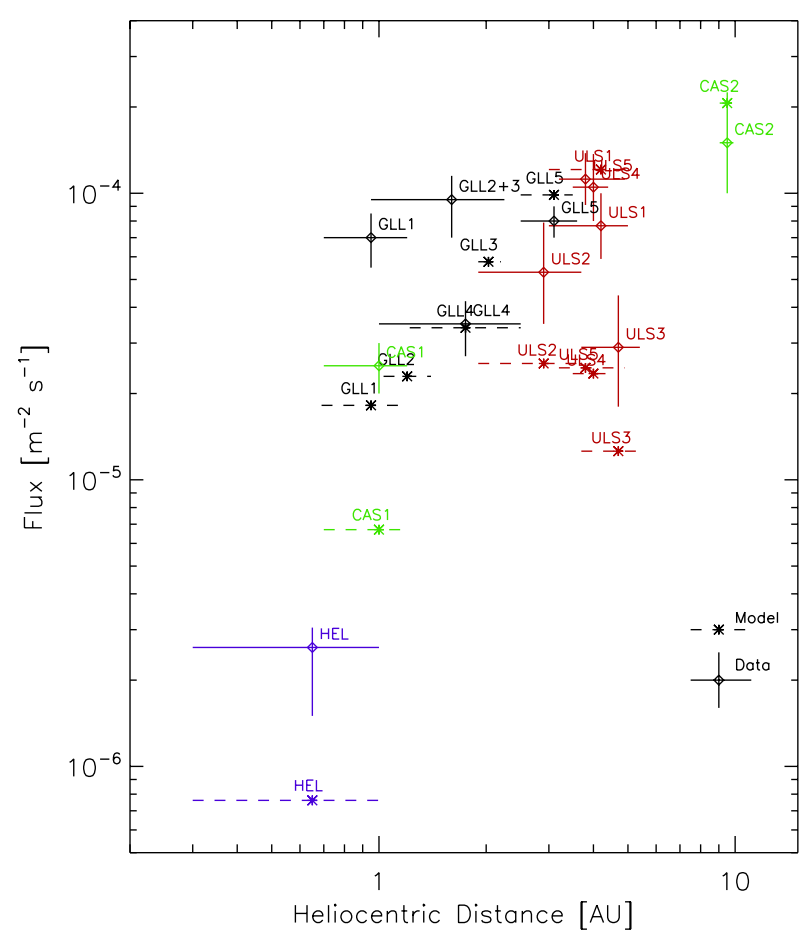

Fig. 6. Dust fluxes simulated with IMEX (asterisks) and measured (diamonds) with Helios (HEL), Galileo (GLL), Cassini (CAS), and Ulysses (ULS). Horizontal bars indicate the distance range where dust measurements and simulations were performed. The detection thresholds and measured particle size ranges listed in Table 1 and shown in Fig. 4 were adopted for the simulations.

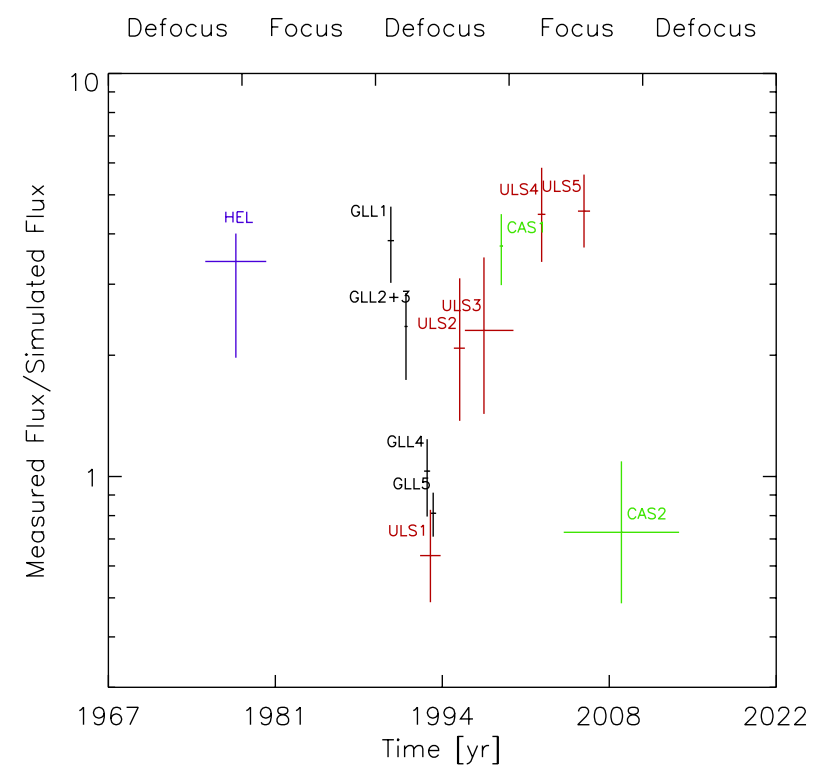

Fig. 7. Ratio of measured to simulated dust fluxes from Fig. 6. Horizontal bars indicate the time intervals when dust measurements and simulations were performed. The modelled focussing and defocussing phases of the IMF in the inner solar system based on observations by the Wilcox Solar Observatory (Hoeksema 2018) are indicated at the top (Strub et al. 2019).

in particular those obtained in spatial regions not used for the model calibration.

All interstellar dust measurements obtained by Helios, Galileo, and Cassini were obtained in the ecliptic plane in environments where solar-system dust populations dominate the particle fluxes. Interplanetary dust particles dominate during all measurement intervals of these missions, while Saturnian dust makes an additional significant contribution in the Cassini 2 interval. Only Ulysses had an ideal configuration for interstellar dust detection far away from the ecliptic plane most of the time. Therefore, the interstellar dust measurements of Helios, Galileo, and Cassini are connected with larger uncertainties in the particle identification and, hence, larger uncertainties in the dust fluxes than those of Ulysses.

For Ulysses the selection criteria for the identification of interstellar particles in the data set used in this work are the same as those that were used by Strub et al. (2015) to analyse the dynamical properties of the particles. Krüger et al. (2015) used different criteria to derive the mass distribution of the particles in order not to induce any bias in the mass distribution. With their technique, these authors identified interstellar particles as small as $2 \times 10^{-18} \mathrm{~kg}$ in the Ulysses data set. This indicates that even though such small particles are strongly filtered by the heliospheric interaction, a fraction of these particles can still reach the inner solar system between 2 and 5 AU.

In Fig. 5 the mass distributions measured by Ulysses are well reproduced by the model. On the other hand, the model overestimates the abundance of small particles close to the detection thresholds for a few of the Galileo measurement intervals and for Helios. In the future we may include these other dust measurements (Galileo, Cassini and Helios) to calibrate the model. This may improve the overall agreement between model and data for the dust fluxes and mass distributions. This is, however, beyond the scope of our present paper.

The Cassini measurements at Saturn (Cassini 2) show a deficit of small particles with masses below approximately $2 \times 10^{-17} \mathrm{~kg}$ (Fig. 5), even though the sensitivity of the instrument enables the detection of smaller particles down to $5 \times 10^{-18} \mathrm{~kg}$ (Altobelli et al. 2016). This can be explained by the filtering of such small particles at the heliopause and the inner heliosphere (Sterken et al. 2013; Slavin et al. 2012); this phenomenon was also observed in the Ulysses interstellar dust data (Landgraf 2000). Furthermore, Altobelli et al. (2016) confirmed the existence of particles with $\beta>1$ with a maximum value reached between $10^{-17}$ and $10^{-16} \mathrm{~kg}$, in good agreement with the $\beta$-mass domains inferred from the Ulysses data (Landgraf et al. 1999; Kimura et al. 2003).

The lack of large interstellar particles in the Cassini 2 data is due to the detection method on the CDA CAT: large impacts, typically micron-sized particles, do not provide time-of-flight spectra with sufficiently well-resolved spectral lines from which the minimum impact speed can be derived. Furthermore, the almost ten times smaller CAT target area compared to the detection area of the Ulysses and Galileo instruments strongly reduces the likelihood of large particle detections.

Figure 6 shows an overall increase in the dust flux as a function of heliocentric distance. The detection thresholds varied for the different missions and measurement intervals, nevertheless this trend illustrates the filtering of the interstellar dust particles by the heliosphere. This confirms the earlier results by Altobelli et al. (2005b) which were based on a smaller data set and on the measurements alone, i.e. without modelling.

Finally, the ratio of measured to simulated flux in Fig. 7 does not show a systematic trend with the solar cycle. It indicates that the description of the heliospheric filtering by the IMF implemented in the model is rather reliable.

The decrease in measured versus simulated flux around 1994 may be caused by the filtering effect of the heliospheric 
boundary which is not yet implemented in the model. This was illustrated by Sterken et al. (2015, their Fig. 19), who found that particles passing through the solar system in 1994 have passed the boundary regions of the heliosphere in the defocusing phase of the solar cycle. In this region, higher particle charges (Kimura \& Mann 1998; Slavin et al. 2012) lead to larger Lorentz forces, thus filtering out interstellar dust pacticles, despite a lower magnetic field strength in comparison with the solar system IMF. While Sterken et al. (2015) suggested this hypothesis to explain the Ulysses data, in this work the Galileo data (GLL4 and GLL5) also seem to follow this trend. Further analysis is needed for confirmation.

The overall agreement between model and data indicates that an extrapolation of the model in space and time should, in general, give reliable predictions for future space missions with a tendency to underestimate the expected dust fluxes. The IMEX model was recently used to study the dust detection conditions for the DESTINY ${ }^{+}$mission, which will measure dust in interplanetary space between 2024 and 2028 and during a dedicated fly-by at the active asteroid (3200) Phaethon (Kawakatsu \& Itawa 2013; Krüger et al. 2019; Kimura et al. 2019; Szalay et al. 2019).

\section{Summary}

We have used the interstellar dust module of the Interplanetary Meteoroid environment for EXploration model (IMEX; Sterken et al. 2013; Strub et al. 2019) to simulate the dynamics of interstellar dust in the solar system. The model covers all relevant forces, i.e. solar gravity, solar radiation pressure, and electromagnetic interaction with the IMF. We compared our model results with in situ interstellar dust measurements obtained with four spacecraft, i.e. Helios, Galileo, Cassini, and Ulysses (Altobelli et al. 2003, 2005a, 2006, 2016; Strub et al. 2015).

Our results can be summarized as follows. The model gives overall good agreement with the spacecraft measurements. Dust fluxes and size distributions simulated for time intervals and spatial regions not covered in the original calibration of the model agree with the in situ spacecraft measurements to within a factor of 2-3. This marks the limit of our current understanding of the interstellar dust flow through the solar system. The model usually underestimates the dust fluxes measured by spacecraft.

A unique time-dependent model, IMEX is designed to predict interstellar dust fluxes and mass distributions for the inner and outer solar system. The model is suited to study dust detection conditions for past and future space missions.

Acknowledgements. The IMEX model was developed under ESA funding (contract 4000106316/12/NL/AF - IMEX). H.K. and P.S. are grateful to the MPI für Sonnensystemforschung and the University of Stuttgart for their support. We are grateful to an anonymous referee whose comments substantially improved the presentation of our results.

\section{References}

Altobelli, N., Kempf, S., Landgraf, M., et al. 2003, J. Geophys. Res., 108, A10 Altobelli, N., Kempf, S., Krüger, H., et al. 2005a, J. Geophys. Res., 110, 7102

Altobelli, N., Kempf, S., Krüger, H., et al. 2005b, in The Spectral Energy Distributions of Gas-Rich Galaxies: Confronting Models with Data, AIP Conf. Proc. 761, 149

Altobelli, N., Grün, E., \& Landgraf, M. 2006, A\&A, 448, 243

Altobelli, N., Dikarev, V., Kempf, S., et al. 2007, J. Geophys. Res., 112, 7105

Altobelli, N., Postberg, F., Fiege, K., et al. 2016, Science, 352, 312

Auer, S. 2001, in Interplanetary Dust, eds. E. Grün, B. A. S. Gustafson, S. F. Dermott, \& H. Fechtig (Berlin: Springer), 385
Baguhl, M., Grün, E., \& Landgraf, M. 1996, Space Sci. Rev., 78, 165

Belheouane, S., Zaslavsky, A., Meyer-Vernet, N., et al. 2012, Sol. Phys., 281, 501

Bertaux, J. L., \& Blamont, J. F. 1976, Nature, 262, 263

Dietzel, H., Eichhorn, G., Fechtig, H., et al. 1973, J. Phys. E Scie. Instrum., 6 , 209

Fechtig, H., Grün, E., \& Kissel, J. 1978, in Cosmic Dust, ed. J. A. M. McDonnell (Chichester: Wiley-Interscience), 607

Frisch, P. C., Dorschner, J., Geiß, J., et al. 1999, ApJ, 525, 492

Göller, J. R., \& Grün, E. 1985, in Properties and Interaction of Interplanetary Dust, eds. R. H. Giese \& P. Lamy (Dordrecht: Reidel), 113

Göller, J. R., \& Grün, E. 1989, Planet. Space Sci., 37, 1197

Grün, E. 1981, Physikalische und chemische Eigenschaften der interplanetaren Staubmessungen des Mikrometeoroidenexperimentes auf Helios, Tech. rep. Bundesministerium für Forschung und Technologie, Forschungsbericht W 81034

Grün, E., Fechtig, H., Hanner, M. S., et al. 1992a, Space Sci. Rev., 60, 317

Grün, E., Fechtig, H., Kissel, J., et al. 1992b, A\&AS, 92, 411

Grün, E., Zook, H. A., Baguhl, M., et al. 1993, Nature, 362, 428

Grün, E., Gustafson, B. E., Mann, I., et al. 1994, A\&A, 286, 915

Grün, E., Baguhl, M., Hamilton, D. P., et al. 1995, Planet. Space Sci., 43, 941

Grün, E., Staubach, P., Baguhl, M., et al. 1997, Icarus, 129, 270

Gustafson, B. A. S. 1994, Ann. Rev. Earth Planet. Sci., 22, 553

Hoeksema, J. 2018, Wilcox Solar Observatory, http://wso.stanford.edu

Kawakatsu, Y., \& Itawa, T. 2013, Adv. Astron. Sci., 146, 12

Kellogg, P. J., Goetz, K., \& Monson, S. J. 2018, J. Geophys. Res., 123, 7211

Kempf, S., Srama, R., Altobelli, N., et al. 2004, Icarus, 171, 317

Kimura, H., \& Mann, I. 1998, ApJ, 499, 454

Kimura, H., \& Mann, I. 1999, in Meteroids 1998, eds. W. J. Baggaley \& V. Porubcan (Slovakia: Astronomical Institute of the Slovak Academy of Sciences), 283

Kimura, H., Mann, I., \& Jessberger, E. K. 2003, ApJ, 583, 314

Kimura, H., Kobayashi, M., Wada, K., et al. 2019, Planet. Space Sci., submitted

Krüger, H., \& Grün, E. 2009, in From the Outer Heliosphere to the Local Bubble, eds. J. Linsky, V. Izmodenov, \& E. Möbius (Heidelberg: Springer)

Krüger, H., Graps, A. L., Hamilton, D. P., et al. 2006, Planet. Space Sci., 54, 919

Krüger, H., Landgraf, M., Altobelli, N., \& Grün, E. 2007, Space Sci. Rev., 130, 401

Krüger, H., Dikarev, V., Anweiler, B., et al. 2010, Planet. Space Sci., 58, 951

Krüger, H., Strub, P., Sterken, V. J., \& Grün, E. 2015, ApJ, 812, 139

Krüger, H., Strub, P., Srama, R., et al. 2019, Planet. Space Sci., 172, 22

Landgraf, M. 1998, Ph.D. Thesis, Ruprecht-Karls-Universität Heidelberg, Heidelberg

Landgraf, M. 2000, J. Geophys. Res., 105, 303

Landgraf, M., Augustsson, K., Grün, E., \& Gustafson, B. A. S. 1999, Science, 286, 319

Landgraf, M., Baggeley, W. J., Grün, E., Krüger, H., \& Linkert, G. 2000, J. Geophys. Res., 105, 343

Landgraf, M., Krüger, H., Altobelli, N., \& Grün, E. 2003, J. Geophys. Res., 108,

Malaspina, D. M., \& Wilson, L. B. 2016, J. Geophys. Res., 121, 9369

Mann, I. 2010, ARA\&A, 48, 173

McComas, D. J., Bzowski, M., Fuselier, S. A., et al. 2015, ApJS, 220, 22

Slavin, J. D., Frisch, P. C., Müller, H.-R., et al. 2012, ApJ, 760, 46

Srama, R. 2009 (Habilitation Thesis), Universität Stuttgart, Stuttgart

Srama, R., Ahrens, T. J. Altobelli, N., Auer, S., et al. 2004, Space Sci. Rev., 114 465

Sterken, V. J., Altobelli, N., Kempf, S., et al. 2012, A\&A, 538, A102

Sterken, V. J., Altobelli, N., Kempf, S., et al. 2013, A\&A, 552, A130

Sterken, V. J., Strub, P., Krüger, H., von Steiger, R., \& Frisch, P. 2015, ApJ, 812,

Sterken, V. J., Westphal, A. J., Altobelli, N., Malaspina, D., \& Postberg, F. 2019, Space Sci. Rev., 69, accepted

Strub, P., Krüger, H., \& Sterken, V. J. 2015, ApJ, 812, 140

Strub, P., Sterken, V. J., Soja, R., et al. 2019, A\&A, 621, A54

Stübig, M. 2002, Ph.D. Thesis, Ruprecht-Karls-Universität Heidelberg, Heidelberg

Swaczyna, P., Bzowski, M., Kubiak, M. A., et al. 2018, ApJ, 854, 119

Szalay, J.-R., Pokorny, P., Horányi, M., et al. 2019, Planet. Space Sci., 165, 194

Westphal, A. J., Stroud, R. M., Bechtel, H. A., et al. 2014, Science, 345, 786

Witte, M., Banaszkiewicz, H., \& Rosenbauer, H. 1996, Space Sci. Rev., 78, 289

Witte, M., Banaszkiewicz, M., Rosenbauer, H., \& McMullin, D. 2004, Adv Space Res., 34, 61

Wolf, H., Rhee, J., \& Berg, O. E. 1976, in Interplanetary Dust and Zodiacal Light, Lecture Notes in Physics, eds. H. Elsässer \& H. Fechtig (Berlin: Springer Verlag), 48165

Wood, B. E., Müller, H.-R., \& Witte, M. 2015, ApJ, 801, 62 